\title{
Apelin/APJ system: A novel therapeutic target for oxidative stress-related inflammatory diseases (Review)
}

\author{
QUN ZHOU ${ }^{1,2^{*}}$, JIANGANG CAO $^{1 *}$ and LINXI CHEN ${ }^{1}$ \\ ${ }^{1}$ Institute of Pharmacy and Pharmacology, Learning Key Laboratory for Pharmacoproteomics, University of South China, \\ Hengyang, Hunan $421001 ;{ }^{2}$ College of Pharmacy, Hunan University of Medicine, Huaihua, Hunan 418000, P.R. China
}

Received September 30, 2015; Accepted March 23, 2016

DOI: $10.3892 / \mathrm{ijmm} .2016 .2544$

\begin{abstract}
Apelin, the endogenous ligand of APJ which is a member of $G$ protein-coupled receptors, has been shown to be expressed in a variety of tissues in vivo and to exert significant biological effects. Studies have indicated that the apelin/APJ system is involved in the regulation of a variety of physiological functions and pathological processes, and that it is associated with cardiovascular diseases (such as atherosclerosis, hypertension, heart failure and myocardial injury), diabetes with microvascular complications, ischemia reperfusion injury, tumors, pre-eclampsia, as well as others. The occurrence of these diseases is closely related to endothelial dysfunction and the local inflammatory response; however, the occurrence of oxidative stress is related to vascular injury, due to the excessive generation of reactive oxygen species (ROS) and can lead to vascular damage and a series of inflammatory reactions. Therefore, this review summarizes the association
\end{abstract}

Correspondence to: Professor Linxi Chen, Institute of Pharmacy and Pharmacology, Learning Key Laboratory for Pharmacoproteomics, University of South China, 28 Changsheng Road, Zhengxiang, Hengyang, Hunan 421001, P.R. China

E-mail: 1xchen6@126.com

${ }^{*}$ Contributed equally

Abbreviations: ROS, reactive oxygen species; VSMCs, vascular smooth muscle cells; ECs, endothelial cells; ICAM-1, intercellular adhesion molecule-1; VCAM-1, vascular cell adhesion molecule-1; MCP-1, monocyte chemoattractant protein-1; NOX, NADPH oxidase; eNOS, endothelial nitric oxide synthase; TNF- $\alpha$, tumor necrosis factor- $\alpha$; L-NAME, NG-nitro-L-arginine methyl ester; DN, diabetic nephropathy; DR, diabetic retinopathy; 8-OHdG, 8-hydroxy-2deoxyguanosine; AOPP, advanced oxidation protein products; PKC, protein kinase C; PARP, poly ADP-ribose polymerase; VEGF, vascular endothelial growth factor; MPTP, mitochondrial permeability transition pore; RISK, reperfusion injury salvage kinase; HIFs, hypoxia inducible factors; PTEN, phosphatase and tensin homolog; TBI, traumatic brain injury; sGC, soluble guanosine cyclase

Key words: apelin, APJ, oxidative stress, reactive oxygen species, inflammation, atherosclerosis, hypertension, ischemia-reperfusion injury between apelin/APJ, oxidative stress and inflammation-related diseases. In addition, drugs targeting the apelin/APJ system are recommended, thus providing a novel therapeutic strategy for oxidative stress-related inflammatory diseases.

\section{Contents}

1. Introduction

2. Apelin induces atherosclerosis by promoting oxidative stress

3 . Apelin induces hypertension by regulating oxidative stress

4. Apelin, oxidative stress and diabetes with associated microvascular complications

5. Apelin regulates oxidative stress by inhibiting ischemiareperfusion injury

6. Apelin regulates oxidative stress, promoting tumor formation and growth

7. Apelin protects the central nervous system by stimulating ROS production

8. Apelin inhibits hypertension in pre-eclampsia by regulating oxidative stress

9. Apelin regulates oxidative stress in the occurrence of other inflammatory diseases

10. Drugs targeting apelin/APJ

11. Conclusions and future prospects

\section{Introduction}

O'Dowd et al firstly discovered a type of receptor protein APJ related to angiotensin type 1 receptor (AT1) in 1993. This was a new G protein-coupled receptor (GPCR) family termed 'orphan receptors', as the relevant endogenous ligand was not found at that time (1). In 1998, Tatemoto et al (2) extracted the endogenous ligand of APJ from the secretions of cattle stomach tissues, and named apelin (APJ endogenous ligand). Recent research has found that ELABELA may be the new endogenous agonist of APJ, and that it plays an important role in cardiac development (3). The amino acid sequence of apelin is similar to that of angiotensin II (Ang II), while APJ and AT1 in the hydrophobic transmembrane region have a homology of $40-50 \%$, which suggests that apelin/APJ may be self-contained and affects the relevant biological function independently $(4,5)$. 
Apelin/APJ has been shown to be expressed in various tissues and cells in the human body, particularly in vascular endothelial cells (ECs) and adipose tissue (6,7). Studies have found that apelin mRNA is expressed in many tissues in rats, such as the heart, kidneys, adipose tissue and the central nervous system [Pope et al (8) and Medhurst et al (9)], which suggests that apelin/APJ can affect the pathophysiological function of the cardiovascular system. The apelin/APJ system has a variety of biological effects. In the cardiovascular system, apelin/ APJ can strengthen cardiac contractility, relax blood vessels and lower blood pressure $(10,11)$. In addition, apelin/APJ can regulate gastrointestinal function and insulin sensitivity, and can promote cell proliferation, migration and angiogenesis, thus regulating immune function (12-16).

Oxidative stress is a condition in which the normal dynamic homeostasis has been overwhelmed by the excessive accumulation of reactive oxygen species (ROS). Oxidative stress is considered to be one of the underlying mechanisms of inflammatory diseases. There are strong links between apelin/APJ and oxidative stress. Apelin is able to suppress the production and release of ROS in adipocytes (17), and to alleviate oxidative stress in cardiomyocytes (18). By contrast, Li et al (19) found that apelin-13 can promote the generation of ROS in vascular smooth muscle cells (VSMCs). Consequently, this review summarizes the role of apelin in regulating oxidative stressrelated inflammatory diseases. Moreover, drugs targeting apelin/APJ are recommended, thus providing a novel therapeutic target for oxidative stress-related inflammatory diseases.

\section{Apelin induces atherosclerosis by promoting oxidative stress}

As discussed below, studies have shown that the development of atherosclerosis (AS) is closely related to VSMC proliferation and the injury of vascular ECs. Moreover, the injury of vascular ECs results in monocytes (MCs) adhering to the ECs, as well as in oxidative stress and inflammation.

Apelin is highly expressed in ECs and VSMCs (20-22). Our laboratory first discovered and reported that the phosphorylation of phosphatidylinositol 3-kinase (PI3K) was stimulated by apelin-13 after binding to APJ, which thereby activated Akt and its downstream signaling molecule, extracellular regulated protein kinase $1 / 2$ (ERK1/2), then promoted the synthesis of cyclin D1, and ultimately induced the proliferation of VMSCs $(23,24)$. Studies have found that apelin may induce the expression of nuclear factor- $\kappa \mathrm{B}(\mathrm{NF}-\kappa \mathrm{B}) / \mathrm{JNK}$ which may then induce intercellular adhesion molecule-1 (ICAM-1), vascular cell adhesion molecule-1 (VCAM-1) and monocyte chemoattractant protein-1 (MCP-1) expression, leading to MCs adhering to human umbilical vein endothelial cells (HUVECs) $(25,26)$.

Oxidative stress and inflammation play a key role in the pathogenesis of AS. Lassègue and Clempus (27) found that NADPH oxidase (NOX) is the main enzyme which generates ROS in blood vessels; ROS produced by the enzyme are involved in the process of occurrence and development of AS. ROS can inhibit the activity of endothelial nitric oxide synthase (eNOS) in cells in order to blunt the production of nitric oxide (NO), while promoting the proliferation of VSMCs and thrombus formation, ultimately leading to or aggravating endothelial dysfunction $(28,29)$. The increase in ROS produc- tion can promote the expression of a variety of inflammatory factors and adhesion molecules in ECs, such as MCP-1 and ICAM-1, VCAM-1, tumor necrosis factor- $\alpha$ (TNF- $\alpha$ ) and interleukin (IL)-1. However, increased inflammatory cytokine production can also promote the increased production of ROS (30). Therefore, oxidative stress promotes the development of AS by regulating the production of ROS.

Li et al (19) found that apelin-13 promotes the increased expression of NOX4, and promotes the generation of ROS. When NOX4 was inhibited, ROS generation and VSMC proliferation induced by apelin- 13 were also inhibited. The phosphorylation of ERK was decreased, indicating that NOX4, as the upstream signaling molecule of ERK, is involved in the promoting effects of apelin-13 on the proliferation of VSMCs. In another study, Li et al (31) also found that apelin-13 promoted the proliferation of VMSCs by activating the ERK-Jagged-1/Notch3-cyclin D1 pathway. Li et al found that apelin-13 promoted the expression of NOX4 and the increased generation of ROS in VSMCs (19), and $\mathrm{Lu}$ et al found that apelin-APJ induced ICAM-1, VCAM-1 and MCP-1 expression through the NF- $\mathrm{kB} / \mathrm{JNK}$ signaling pathway (26). Therefore, we speculated that apelin and oxidative stress may be involved in the development of AS through the NOX4-ROS-NF- $\mathrm{kB}$ /ERK signaling pathway (Fig. 1).

Both apelin and oxidative stress can regulate the development of AS; we thus hypothesized that there is an association between apelin and oxidative stress. In 2007, Hashimoto et al reported that AS plaques and NOX were markedly decreased in the vascular tissue of APJ receptor and apolipoprotein E (apoE) double knockout mice; thus, the authors suggested that the APJ receptor is involved in the development of AS through oxidative stress (32). In 2009, Leeper et al (33) demonstrated that the apelin/ APJ system is a regulatory factor of vascular oxidative stress. In a rat model of AS induced by a high-cholesterol diet (32), apelin/APJ was shown to regulate oxidative stress in vascular tissue, suggesting that apelin is a key factor in the process of AS caused by a high cholesterol diet; APJ deficiency can protect blood vessels from generating oxidative stress-related AS, and can also prevent oxidative stress-induced AS (34). In conclusion, apelin and oxidative stress can promote the development of AS, but a deficiency in APJ can prevent the development of AS due to oxidative stress.

\section{Apelin induces hypertension by regulating oxidative stress}

Apelin can regulate angiogenesis and adjust blood pressure through different mechanisms. A previous study demonstrated the anti-hypertensive effects of apelin; the administration of apelin-13 to male Wistar rats via intravenous injection reduced systolic and diastolic blood pressure temporarily (35). The main mechanism responsible for the reducing effect of apelin on blood pressure is through eNOS phosphorylation, promoting the release of $\mathrm{NO}$, and thereby promoting endothelial-dependent vasodilation. As previously demonstrated, following an intraperitoneal injection of apelin-12, apelin-13 and apelin-36 to anesthetized rats, the mean arterial pressure decreased, and the results revealed that the anti-hypertensive effects of apelin-12 were the most prominent. Following the exogenous apelin-12 injection into mice, the nitrite levels in plasma transiently increased. However, in rats treated with the eNOS inhibitor, NG-nitro-L-arginine methyl ester (L-NAME), prior to the 


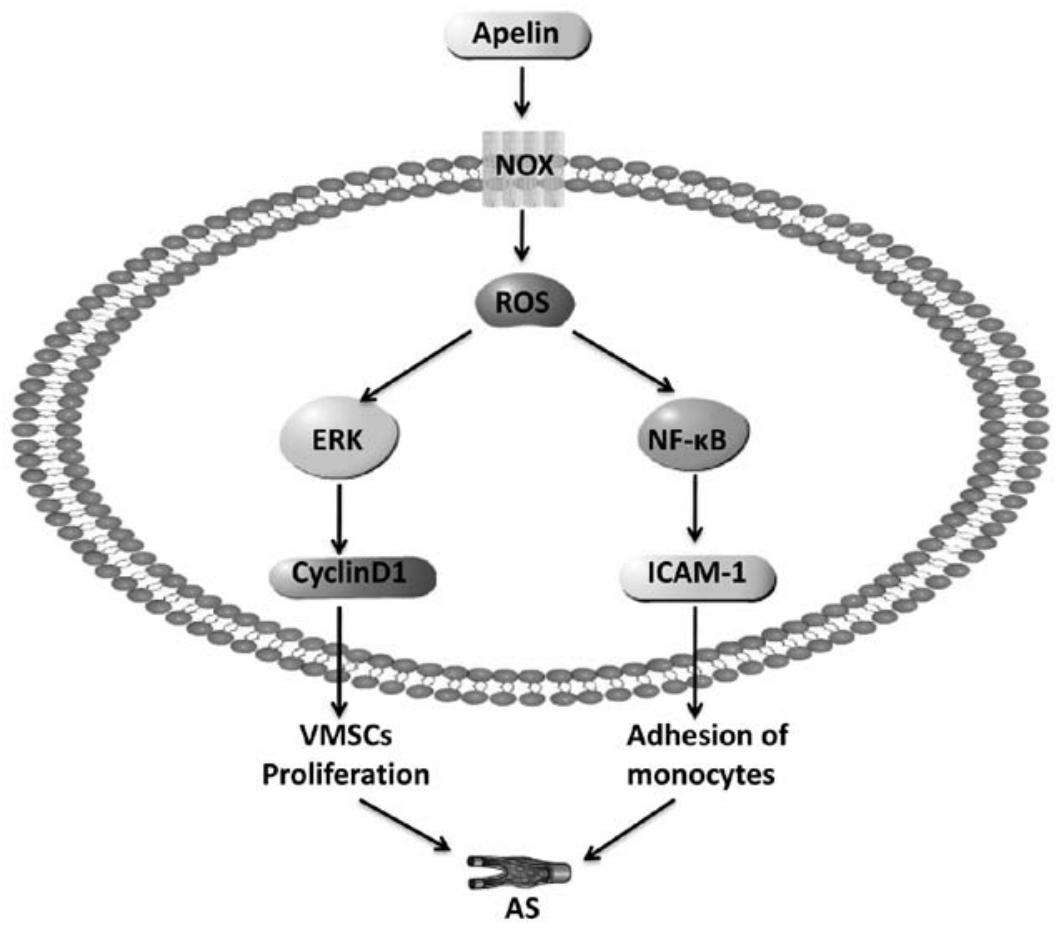

Figure 1. Apelin induces the generation of ROS and promotes the formation of AS. $\downarrow$, promotion. AS, atherosclerosis; NOX, NADPH oxidase; ROS, reactive oxygen species; ERK, extracellular regulated protein kinases; NF-kB, nuclear factor-kB; VCAM-1, vascular cell adhesion molecule-1.

injection of apelin-12, no significant change in blood pressure was observed; thus the anti-hypertensive effects of apelin rapidly diminished $(10,36)$. Japp et al $(37)$ found that the acute administration of apelin to humans leads to human peripheral vascular and coronary vasodilation and increases cardiac output. In APJ-deficient mice injected with apelin, there was no significant change in blood pressure (10), suggesting that apelin needs to develop the function of vasodilation and lower blood pressure through its APJ receptors. Katugampola et al (20) found that in the saphenous vein in vitro, apelin combined with the APJ receptor in vascular smooth muscle, and stimulated myosin light chain to induce vascular contraction, indicating that apelin may directly constrict vascular smooth muscle; however, only in the intact endothelium there was a vasodilatory effect when combining apelin with endothelial APJ. The apelin/APJ system is involved in the regulation of blood pressure through a variety of mechanisms, and has a certain connection with the condition that depends on whether the endothelium is intact or not.

The occurrence of eNOS uncoupling is caused by oxidative stress (38). L-arginine (L-Arg) as the substrate generated by eNOS in vivo and tetrahydrobiopterin (BH4) as the co-factor in short supply, may lead to eNOS dysfunction and the production of $\mathrm{O}_{2}^{-}$, but not $\mathrm{NO}$, so as to promote the development of hypertension and the occurrence of vascular complications. ROS are superoxide anions, and can result in the inactivation of NO, which is an endothelium-derived relaxing factor, further leading to detrimental effects on vasodilation, leading to endothelial dysfunction, and finally giving rise to hypertension (39). Thus, oxidative stress is involved in the occurrence of hypertension and has a close association with endothelial dysfunction.

The incidence of hypertension is closely associated with a significant increase in ROS generation in the human body.
The elevated levels of ROS may be associated with the occurrence of oxidative stress, directly resulting in vascular injury and a series of inflammatory reactions, finally causing hypertension. Thus, the aforementioned apelin- 13 can promote the increased expression of NOX4, and can also promote the generation of ROS. The NADH/NADPH oxidative system of the vascular wall can be regulated by Ang II, which can markedly increase the generation of $\mathrm{O}_{2}{ }^{-}$and $\mathrm{H}_{2} \mathrm{O}_{2}$, causing vascular injury, increased tension and increased resistance, resulting in vascular remodeling, and ultimately in elevated blood pressure. Moreover, in the pathogenesis of hypertension, the renin angiotensin system (RAS) plays an important role. Ang II is known as one of the strongest vasoconstrictor substances, when acting on vascular smooth muscle, it can cause the arteriole contracts of the whole body to increase arterial pressure; under physiological and pathological conditions, apelin/APJ has completely opposite effects to the function of RAS; thus, under such conditions, apelin has an antagonistic effect to that of Ang II $(40,41)$. In conclusion, it is suggested that apelin can increase hypertension by regulating the NOX4-Ang II pathway. Moreover, apelin can also inhibit the oxidative stress which is involved in the occurrence of hypertension, possibly through the eNOS/NO pathway (Fig. 2).

\section{Apelin, oxidative stress and diabetes with associated microvascular complications}

Some research results have indicated that apelin has a close association with diabetic microvascular complications, such as diabetic nephropathy (DN), diabetic retinopathy (DR) and pathological changes of the nervous system. Oxidative stress is also thought to be one of the underlying mechanisms of diabetic microvascular complications (42). 


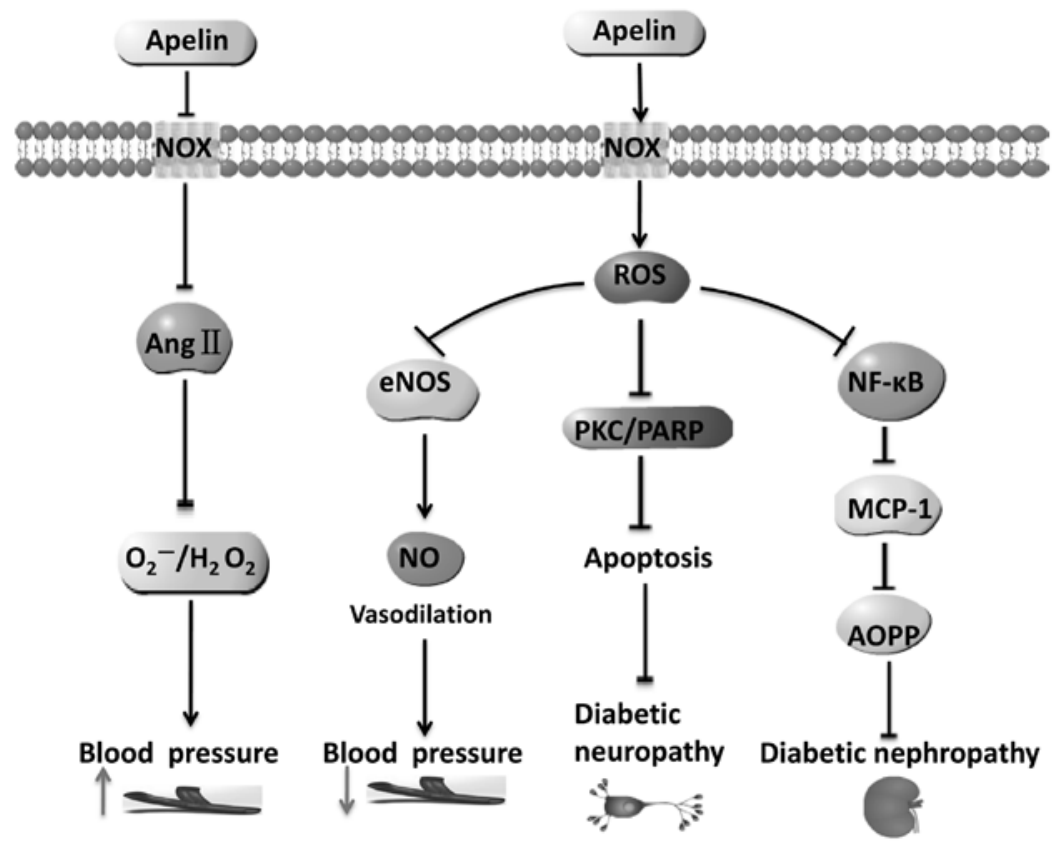

Figure 2. Apelin induces ROS generation, participating in the occurrence of hypertension and diabetes-related microvascular complications. $\downarrow$, promotion; $\perp$, inhibition; $\uparrow$ (gray), up; $\downarrow$ (gray), down. ROS, reactive oxygen species; eNOS, endothelial nitric oxide synthase; PKC, protein kinase C; PARP, poly ADPribose polymerase; MCP-1, monocyte chemoattractant protein-1; AOPP, advanced oxidation protein products.

Glomerular capillary damage directly leads to the occurrence of DN; the increase in free radicals and oxidative stress is one of the causes of DN (43). Furthermore, the inflammatory pathway plays an important role in the pathogenesis of DN. 8-Hydroxy-2-deoxyguanosine $(8-\mathrm{OHdG})$ is the specific and main product of DNA oxidative damage, and advanced oxidation protein products (AOPP) reflects the protein damage caused by oxidative stress. The levels of AOPP in plasma and $8-\mathrm{OHdG}$ in the urine of patients with DN with large amounts of albumin in urine were significantly higher than those of the patinets with normal albumin urine levels (44). The data suggest that oxidative stress promotes the development of DN. Hyperglycemia activates NF- $\mathrm{kB}$ through ROS to promote the expression of MCP-1; the expression of MCP-1 in renal tissue is a marker of the inflammatory occurrence $(45,46)$.

Studies have shown that the subcutaneous injection of apelin can be effective in preventing the occurrence of renal enlargement, reducing the expression of MCP-1 and VCAM-1, and inhibiting the activation of NF- $\mathrm{KB}$ (47). Apelin can also restore the levels of antioxidant enzymes in the kidneys of diabetic mice, reduce oxidative stress, and prevent damage to kidneys from oxidative stress (48). Thus, apelin may be a therapeutic target in DN by exerting anti-oxidantive effects (Fig. 2).

An important reason for the occurrence of diabetic neuropathy is the increased formation of advanced glycation end-products caused by oxidative stress, promoting the apoptosis of nerve cells mediated through the phosphoinositide/ protein kinase $\mathrm{C}(\mathrm{PKC})$ system and DNA repair enzyme poly ADP-ribose polymerase (PARP), NF- $\mathrm{KB}$ and damage sensory fibers $(49,50)$. Studies have demonstrated that apelin inhibits the activation of NF- $\mathrm{kB}$ (47), and thus protects nerve cells. In cortical neurons, apelin can also inhibit the generation of ROS (51). Thus, apelin can inhibit oxidative stress to prevent the occurrence of diabetic neuropathy.
DR is the leading cause of human blindness. The close associatoin between vascular endothelial growth factor (VEGF) and proliferative DR is considered to be the most important mechanism responsible for DR (52). Although the inhibition of VEGF may reduce retinal neogenesis, it cannot completely inhibit the formation of neovascularization and the proliferation of retinal cells caused by ischemia. Studies have shown that the mRNA levels of apelin, APJ and VEGF are all increased in the vascular tissue membrane in proliferative DR (53), and that VEGF is involved. It has been proven that apelin promotes the expression of VEGF (54). Saint-Geniez et al (55) found that apelin/APJ is involved in retinal angiogenesis in mice, and has a unique expression pattern. Thus, the function of apelin in promoting retinal neogenesis is considered to be one of the causes of DR. Studies have indicated that oxidative stress has a significant association with the occurrence of DR. The enhancement of free radical activity and the decrease in antioxidant capacity are the important mechanisms responsible for DR. Increased levels of oxygen free radical in vivo leads to an increase in retinal cell apoptosis, as oxygen free radicals can activate the caspase family through the mitochondrial pathway, leading to the increased concentration of intracellular $\mathrm{Ca}^{2+}$ in retinal cells, and inducing apoptosis through the activation of NF- $\mathrm{\kappa B}(56,57)$. Thus, apelin and oxidative stress are involved in the occurrence of DR, and the inhibition of the apelin/APJ system in the eyes may be an effective method to prevent DR (Fig. 2).

\section{Apelin regulates oxidative stress by inhibiting ischemia- reperfusion injury}

During the process of ischemia-reperfusion injury, superoxide anion $\left(\mathrm{O}_{2}^{-}\right)$synthesis occurs. In addition to superoxide anion, ROS also play an important role in ischemia-reperfusion injury. It has been suggested that oxidative stress may have a 


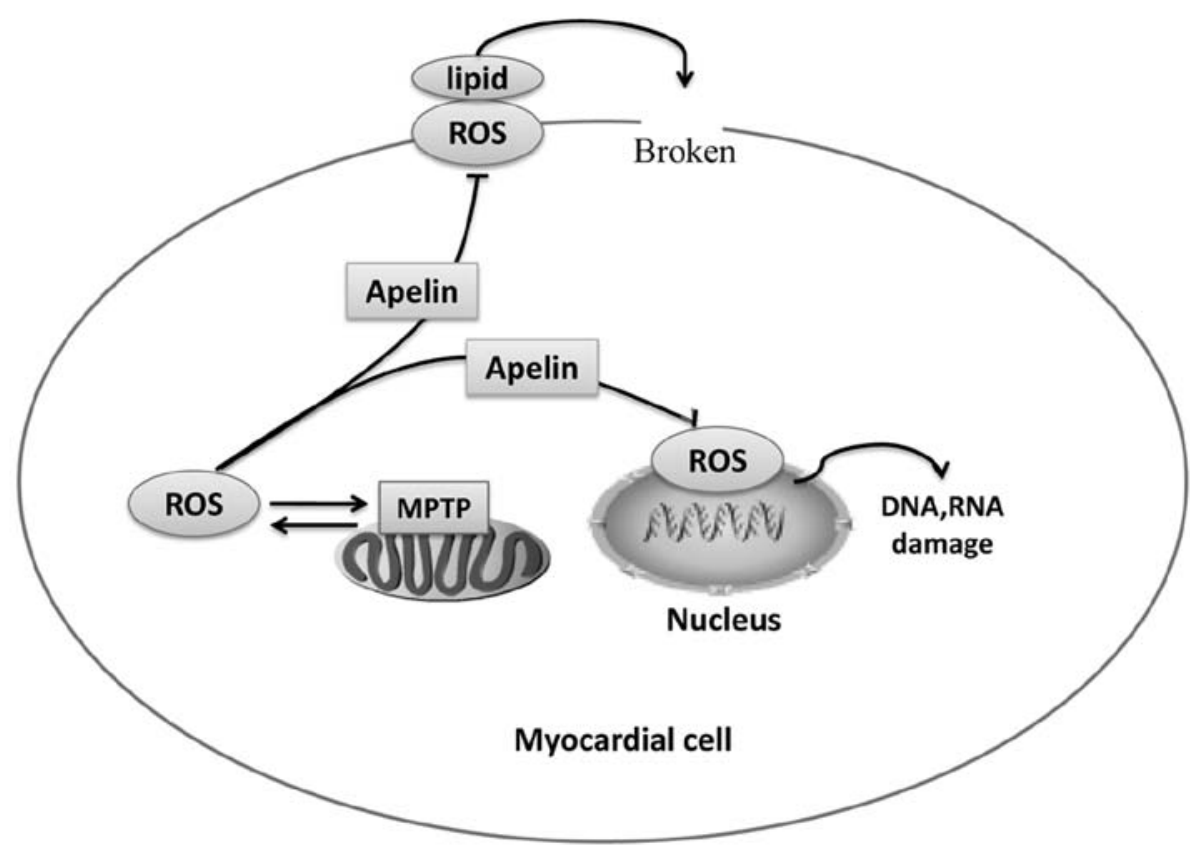

Figure 3. The main mechanism of of action of apelin is to inhibit oxidative stress, regulating ischemia-reperfusion injury. $\downarrow$, promotion; $\perp$, inhibition; MPTP, mitochondrial permeability transition pore.

definite association with ischemia-reperfusion injury. The main mechanisms of the regulation of ischemia-reperfusion injury by oxidative stress are the functional inactivation of intracellular DNA, RNA proteins and other substances so as to lead to cell dysfunction (58-60), mainly through ROS: the overload of ROS promotes mitochondrial permeability transition pore (MPTP) opening, which in turn increases the release of mitochondrial ROS, to form a vicious positive feedback loop between ROS activation and ROS release. ROS, in combination with lipids can damage cell membranes and organelle membranes in myocardial cells, reducing membrane fluidity and permeability; ROS can also attack the genetic material to cause the cross linking or breaking of DNA and RNA, affecting gene expression in myocardial cells (Fig. 3).

During the process of ischemia-reperfusion, apelin can protect myocardial cells against oxidative stress, and reduce the damage to myocardial cells (Fig. 3). Zeng et al (61) found that ROS levels in myocardial cells increased following exposure to hypoxia, and the addition of apelin to the medium $30 \mathrm{~min}$ prior to exposure to hypoxia effectively reduced the levels of ROS. It has been shown that apelin can activate eNOS and enhance NO release through reperfusion injury salvage kinase (RISK), and may thus inhibit mitochondrial oxidative damage and lipid peroxidation (62). All the above-mentioned results strongly suggest that apelin plays an antagonistic role in the process of ischemia-reperfusion, thus exerting a myocardial protective effect.

\section{Apelin regulates oxidative stress, promoting tumor formation and growth}

With the continuous progress in relevant research, the function of apelin and oxidative stress in tumors has attracted increasing attention. In 2007, Sorli et al (63) firstly reported that hypoxia caused by the hypermorphosis of tumor cells can promote the expression level of apelin. The hypoxia-induced elevation in apelin expression is most likely mediated by hypoxia inducible factors (HIFs) (64). Under a hypoxic environment, increased levels of ROS induce the expression of HIF in cancer stem cells (CSCs) (65). ROS are also closely related to tumorigenesis, and thus it is hypothesized that oxidative stress induced by ROS is closely associated with tumorigenesis and may be regulated by apelin. Studies have indicated the involvement of oxidative stress in the formation and development in bladder cancer, and there are observed changes in the activity of transcription factors, such as hypoxia-inducible factor HIF-1 $\alpha$ (66). Raina et al (67) evaluated the efficacy of grape seed extract (GSE) in bladder cancer and found that GSE-generated oxidative stress induced marked programmed cell death in human bladder cancer cells.

Although ROS are involved in tumorigenesis, they also play a role in the treatment of tumors (68), and the mechanisms involved include DNA damage induced by ROS. More importantly, lipid peroxidation induced by ROS and damage to DNA or proteins can cause or promote the development of tumors. Naturally, oxidative stress can stimulate tumorigenesis and growth, but can also inhibit tumor progression. Apelin can inhibit the generation of ROS, which suggests that apelin suppresses tumorigenesis by regulating oxidative stress. However, studies have found that cancer stem cells, as a type of cell with the characteristics of stem cells in tumor tissues, can resist the inhibitory effects of oxidative stress caused by radiotherapy or chemotherapy (69-71). There is evidence that compared with ordinary tumor cells, there are fewer oxidation products of DNA, stronger resistance to oxidative stress, and higher activation level of the antioxidant stress system, existing in cancer stem cells (71).

The above-mentioned studies all demonstrate that apelin and oxidative stress can cause or accelerate tumorigenesis and growth; apelin and ROS are possible targets for the diagnosis and treatment of tumors, and for the prediction of prognosis. 
However, the specific molecular mechanisms of action of apelin and oxidative stress require further investigation.

\section{Apelin protects the central nervous system by stimulating ROS production}

A recent study demonstrated that apelin-36 reduced the cerebral infarct volume and promoted long-term functional recovery following hypoxic/ischemic (H/I) brain injury. The mechanisms responsible for the protective effects of apelin-36 against $\mathrm{H} / \mathrm{I}$ brain injury involved a decrease in the levels of cleaved caspase- 3 and Bax. Apelin-36 also increased the expression level of phosphorylated Akt; however, with the use of a specific PI3K inhibitor, the levels of phosphorylated Akt decreased, and the protective effects of apelin-36 against apoptosis were attenuated (72). This suggests that the PI3K/Akt pathway is at least partly involved in the anti-apoptotic effects of apelin-36. Thus, apelin-36 may be a promising therapeutic agent in the treatment of ischemic brain injury. As apelin can promote the phosphorylation of PI3K/Akt and simulate the expression of NOX-ROS, PI3K/Akt may thus also be associated with ROS. Silva et al (73) demonstrated that ROS mediate the phosphatase and tensin homolog (PTEN)-independent activation of the PI3K/Akt pathway in some $\mathrm{T}$ cell acute lymphoblastic leukemia (T-ALL) cells. Min et al suggested that ROS may activate PI3K/Akt and Nrf2 signaling (74). Thus, it can be hypothesized that apelin induces ROS production, activates PI3K/Akt, and ultimately, exerts anti-apoptotic and neroprotective effects.

Apelin-13 can also protect against serum deprivationinduced apoptosis, as apelin-13 not only blocks typical apoptosis, but also exerts inhibitory effects on the NMDA-induced increase in intracellular $\mathrm{Ca}^{2+}$ levels and excitotoxicity (51). The study of Khaksari et al (75) demonstrated that apelin-13 protects the brain against ischemia-reperfusion injury and cerebral edema. In addition, apelin-13 attenuates traumatic brain injury (TBI)-induced brain damage by suppressing autophagy (76). Autophagy may be involved in the protective effects of apelin-13 against damage to neurons in TBI. Another study found that apelin expectedly had a neuroprotective effect combined with VEGF, but not alone (77). It is clear that apelin, which activates the PI3K/Akt signaling pathway, enhances angiogenesis induced by VEGF (78). All the above-mentioned data indicate that apelin can protect the brain against ischemiareperfusion injury, and the mechanisms involved may be associated with the activation of the PI3K/Akt pathway and ROS.

\section{Apelin inhibits hypertension in pre-eclampsia by regulating oxidative stress}

Pre-eclampsia is a hypertensive disorder and a complication of some pregnancies. Studies have shown that apelin is also closely associated with normal pregnancies and pre-eclampsia. Compared with normal pregnancies, the RNA and protein level of apelin are significantly decreased in pre-eclamptic placentas $(79,80)$. The above findings suggest that the reduced expression of apelin may be associated with preeclampsia and the apelinergic system plays an important role in preeclampsia-induced hypertensive maternal disorders. A study on pregnancy-induced hypertension $(\mathrm{PIH})$ indicated that
apelin/APJ was poorly stained in the ECs of early-onset PIH placentas, thus reflecting poor fetal growth (81).

It has been shown that $\mathrm{NO}$ signaling is important for placental function, and in particular for the maintenance of vascular tone. In the normal placenta, the release of NO depends on the availability of L-Arg, and when L-Arg bioavailability is reduced, the synthesis of NO is inhibited, leading to rapid NO degradation by ROS, for example $\mathrm{O}_{2}$. Downstream NO in smooth muscle cells during pregnancy, then activates the NO sensitive soluble form, soluble guanosine cyclase (sGC), which is responsible for cGMP generation, then activates cGMP-sensitive protein kinase $\mathrm{G}$ (PKG), and subsequently promotes vasodilation (82). Consistent with laboratory research results, apelin promotes the phosphorylation of PI3K/Akt, and then activates eNOS, inducing the generation of NO. However, in pre-eclampsia, the expression of a group of GPCRs (group I) is reduced, and the L-Arg availability for NOS may be further minimized, which leads to the aberrant and rapid NO degradation by ROS, subsequently, inducing vasoconstriction through the NO-cGMP-PKG pathway. Moreover, apelin inhibits ROS formation. Above all, apelin regulates oxidative stress, inhibiting hypertension through NO-cGMP pathway in pre-eclampsia (Fig. 4).

\section{Apelin regulates oxidative stress in the occurrence of other inflammatory diseases}

In intestinal inflammation, a variety of inflammatory cytokines can upregulate apelin expression by activating the Jak/STAT3 pathway; apelin upregulation is able to promote the proliferation of epithelial cells $(64,83)$. The incidence of inflammatory bowel disease is closely related to the oxidative stress generated by intestinal epithelia. The incidence of oxidative stress can cause intestinal epithelial cell damage; ROS can induce the activation of a variety of signaling pathways to promote intestinal epithelial cell apoptosis, including the PI3K pathway, which plays an important role in this process $(84,85)$. The Jak/STAT3 and PI3K/Akt pathways are closely associated with the IL-6 receptor family; thus, it can be hypothesized that apelin/oxidative stress promotes the occurrence and development of intestinal inflammation, and may be related to the IL-6 receptor family.

Septicopyemia is a syndrome of systemic inflammatory response caused by infection. Research has shown that apelin expression is closely related to septicopyemia. During the occurrence of septicopyemia, the levels of apelin expression in serum are not only significantly higher compared with the levels prior to the occurence of septicopyemia; however, the increase in apelin expression can antagonize myocardial cell injury from septicopyemia (86-88). Oxidative stress and inflammation are closely related to septicopyemia (89). During the course of septicopyemia, the electron transfer of mitochondrial respiratory chain can produce ROS which then leads to oxidative stress (90). The electron transfer causes myocardial damage directly; oxidative stress can also result in cell death (91). However, in this process, lipid peroxidation is significantly increased. Apelin, as a new-type peptide, has a protective function, and can attenuate damage to heart tissue and reduce lipid peroxidation (92). Therefore, apelin can be used for the diagnosis and treatment of septicopyemia as a promising therapeutic agent (93), and can prevent myocardial injury caused by oxidative stress in the process of septicopyemia. 


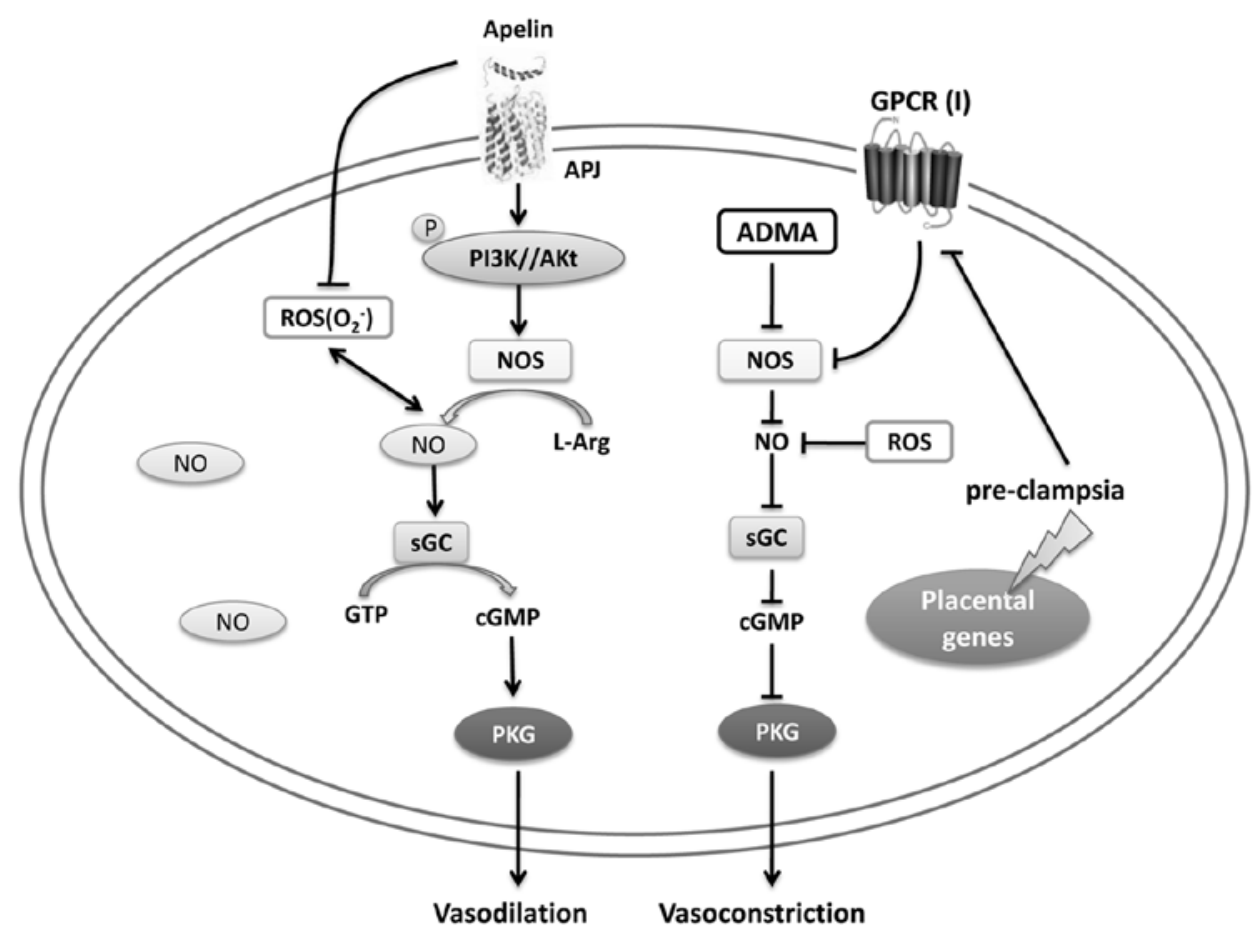

Figure 4. Apelin regulates oxidative stress, modifying vascular tone in pre-eclampsia. $\downarrow$, promotion; $\perp$, inhibition. GPCR, G protein coupled receptor; ADMA, symmetric dimethylarginine; PKG, protein kinase G.

Table I. Function of the apelin/APJ system in inflammation-related diseases.

\begin{tabular}{|c|c|c|c|c|c|}
\hline Disease types & Experiment models & Treatment & Pathway & Function & Authors/(Refs.) \\
\hline AS & VSMCs & Exogenous apelin & ERK1/2, PI3K/Akt & Exacerbation & $\begin{array}{l}\text { Li et al (23) } \\
\text { Liu et al (24) }\end{array}$ \\
\hline AS & HUVECs & Exogenous apelin & NF- $\kappa \mathrm{B} / \mathrm{JNK}$ & Exacerbation & Lu et al (26) \\
\hline Hypertension & Wistar rats & Exogenous apelin & eNOS/NO & Inhibition & Lee et al (35) \\
\hline DN & Ove26 mice & Exogenous apelin & $\mathrm{NF}-\kappa \mathrm{B}$ & Inhibition & Day et al (47) \\
\hline DR & Diabetic rats & Exogenous apelin & VEGF & Exacerbation & Lu et al (54) \\
\hline I/R injury & Rat heart models & Exogenous apelin & ROS & Cardioprotection & Zeng et al (61) \\
\hline Tumor & TS/A cells & Apelin gene & Gaseous hypoxia & Exacerbation & Sorli et al (63) \\
\hline $\mathrm{H} / \mathrm{I}$ brain injury & $\mathrm{H} / \mathrm{I}$ brain injury models & Exogenous apelin & PI3K/Akt & Neuroprotection & Gu et al (72) \\
\hline $\begin{array}{l}\text { Intestinal } \\
\text { inflammation }\end{array}$ & Rat ileum & Enteric apelin & Jak/STAT3 & Exacerbation & Han et al (83) \\
\hline Pyemia & Male rats & Exogenous apelin & $\begin{array}{l}\text { Inhibit MCP-1 } \\
\text { and IL-8 }\end{array}$ & Cardioprotection & Pan et al (88) \\
\hline
\end{tabular}

DN, diabetic nephropathy; DR, diabetic retinopathy; I/R injury, ischemia reperfusion injury; H/I brain injury, ischemic brain injury; VSMCs, vascular smooth muscle cells; HUVECs, human umbilical vein endothelial cells; H/I brain injury models, ischemic brain injury models; TS/A cells, TS/A mammary carcinoma cells; MCP-1, monocyte chemoattractant protein-1; IL-8, interleukin-8; PI3K, phosphatidylinositol 3-kinase; ERK1/2, extracellular regulated protein kinases $1 / 2 ; \mathrm{NF}-\mathrm{\kappa B}$, nuclear factor- $\mathrm{\kappa B}$.

\section{Drugs targeting apelin/APJ}

The above-mentioned research results demonstrate that the apelin/APJ system plays an important role in the process of occurrence and development of various diseases (AS, hypertension, diabetes, etc.). Therefore, the apelin/APJ system has become one of the potential drug targets (94), and drugs targeting aplelin/APJ may apply to the treatment of several diseases.
Recent studies have reported small molecule agonists and antagonists targeting APJ. E339-3D6 was the firstly reported non-peptide agonist of APJ receptor which can inhibit the release of antidiuretic hormone of water-dependent induction in rats and reduce arterial blood pressure (95). ML233, as another small molecule compound ligand of APJ, can selectively inhibit AT1 receptors, which can promote vasoconstriction through phospholipase C (96). Both E339-3D6 and ML233 can inhibit 
the release of forskolin-activated rennin induced by the cAMP pathway, which can play an important role in the development of hypertension (97).

In addition, studies on APJ receptor antagonists have also made rapid progress. 4-Oxo-6-((pyrimidin-2-ylthio)methyl)4H-pyran-3-yl4-nitrobenzoate (ML221) is the first APJ receptor antagonists to be found, primarily as tool drug used to mark APJ receptor (98); later, ALX40-4C as another APJ receptor antagonists has also been found. ALX40-4C is the APJ and CXCR4 receptor antagonists containing nine arginine residues; the drug by combing with APJ receptor can inhibit ligand-induced intracellular calcium mobilization and receptor internalization, in order to achieve the effect of lowering blood pressure (99). Above the small molecules, a number of peptides based on apelin-13 has led to development of more potent and stable analogs targeted APJ (100). As showed in the review of Cao et al (94), the analogs of apelin may directly reduce blood pressure or by activating Akt-eNOS/NO pathway.

Apelin could regulate the oxidative stress-linked inflammation diseases, so the drugs targeted apelin may apply to the treatment the diseases. Studies showed that crude drug puerarin could downregulated expression of apelin and exerted a protective effect on renal hypertension (101), suggesting that puerarin may cure oxidative stress-linked blood pressure. In conclusion, every drugs targeted APJ and apelin, they play an important role in the research of pharmacological effect on Apelin/APJ system, and also become the reliable tools to explore the system on the development mechanism of oxidative stress-mediated disease.

\section{Conclusions and future prospects}

Studies have indicated that the apelin/APJ system plays a certain role in inflammation-related diseases (Table I). Apelin/ APJ regulates oxidative stress in the occurrence of inflammation-related diseasesl; however, this process is intricate. Thus, further research into this matter is required.

At a first glance, the effects of apelin and oxidative stress are similar. On the one hand, vascular endothelial shear stress, oxidative stress, inflammation and other factors can alter apelin expression; on the other hand, apelin has an impact on insulin sensitivity and cardiovascular function, exerting anti-inflammatory and antioxidant effects, acting as an adipokine (102). In addition, the overproduction of ROS can lead to a series of inflammatory reactions, and apelin can regulate the production of ROS; thus,there is a close link between apelin, oxidative stress and inflammation.

In addition, apelin regulates oxidative stress-related inflammatory diseases. However, there are still certain contradictions and doubts. For example, the assumption that apelin inhibits ROS is opposite to the conclusion made by our research group; apelin and oxidative stress have an opposite effect on hypertension; the effects of apelin and oxidative stress on tumorigenesis are not yet fully elucidated; apelin and oxidative stress promote the occurrence and development of intestinal inflammation, and this is related to the IL-6 receptor family. Thus, these contradictory effects may be caused by the existence of multiple isoforms of apelin (103), the activation of diverse signaling pathways and different stimuli/factors. For example, pyr-apelin-13 can reduce renal enlargement and inflammation, and improve albumin urine levels in DN (47); however, there are also studies showing that the angiogenic effects of apelin may hinder or blunt its beneficial effects on albumin urine levels (104). Thus, the regulatory effects of apelin regulates on oxidative stress-related diseases warrant further investigation.

Finally, the apelin/APJ system is abundantly found, which suggests that apelin is a critical factor in the occurrence of various diseases. The apelin/APJ system has a number of biological functions; for instance, apelin has been shown to exert cardioprotective effects in renal ischemia-reperfusion injury (105). In cerebral ischemia-reperfusion, both apelin-13 and apelin-36 can protect neurons and inhibit damage induced by inflammation by activating the PI3K/Akt pathway $(72,106,107)$. Furthermore, small molecular compounds may be developed to target the apelin/APJ system for the treatment of inflammationrelated diseases.

In summary, in this review, we discussed the related functions of the apelin/APJ system, and its effects on oxidative stress and inflammation-related diseases, and provide a new theoretical foundation for the study of the occurrence and development of oxidative stress-related inflammatory diseases. The apelin/APJ system functions as a double-edged sword in regulating oxidative stress-related inflammatory diseases. With the continuous progress made by research, an increasing number of apelin/APJ functions are being discovered. Thus, the apelin/APJ system may prove to be a novel therapeutic target in inflammation-related diseases. Further research is warranted however, in order to further elucidate the different apelin subtypes and their related functions. Consequently, the relevant mechanisms of action of apelin/APJ and its role in oxidative stress or its antioxidant effects in inflammation-related diseases need to be further explored in future studies.

\section{Acknowledgements}

This study was supported by grants from the National Natural Science Foundation of China (nos. 81270420 and 81470434), the Hunan Province Cooperative innovation Center for Molecular Target New Drug Study [Hunan Provincial Education Department document (approval no. 2014-405)] the Hunan Provincial Natural Science Foundation of China (no. 14JJ3102), the project funded by China Postdoctoral Science Foundation (no. 2014M560647).

\section{References}

1. O'Dowd BF, Heiber M, Chan A, Heng HH, Tsui LC, Kennedy JL, Shi X, Petronis A, George SR and Nguyen T: A human gene that shows identity with the gene encoding the angiotensin receptor is located on chromosome 11. Gene 136: 355-360, 1993.

2. Tatemoto K, Hosoya M, Habata Y, Fujii R, Kakegawa T, Zou MX, Kawamata Y, Fukusumi S, Hinuma S, Kitada C, et al: Isolation and characterization of a novel endogenous peptide ligand for the human APJ receptor. Biochem Biophys Res Commun 251: 471-476, 1998.

3. Xie F, Lv D and Chen L: ELABELA: A novel hormone in cardiac development acting as a new endogenous ligand for the APJ receptor. Acta Biochim Biophys Sin (Shanghai) 46: 620-622, 2014.

4. Lee DK, Cheng R, Nguyen T, Fan T, Kariyawasam AP, Liu Y, Osmond DH, George SR and O'Dowd BF: Characterization of apelin, the ligand for the APJ receptor. J Neurochem 74: 34-41, 2000 . 
5. Saavedra JM, Correa FM, Seltzer A, Pinto JE, Viglione P and Tsutsumi K: Enhanced angiotensin converting enzyme binding in arteries from spontaneously hypertensive rats. J Hypertens 10: $1353-1359,1992$

6. Choe W, Albright A, Sulcove J, Jaffer S, Hesselgesser J, Lavi E, Crino P and Kolson DL: Functional expression of the seven-transmembrane HIV-1 co-receptor APJ in neural cells. J Neurovirol 6 (Suppl 1): S61-S69, 2000.

7. Habata Y, Fujii R, Hosoya M, Fukusumi S, Kawamata Y, Hinuma S, Kitada C, Nishizawa N, Murosaki S, Kurokawa T, et al: Apelin, the natural ligand of the orphan receptor APJ, is abundantly secreted in the colostrum. Biochim Biophys Acta 1452: 25-35, 1999.

8. Pope GR, Roberts EM, Lolait SJ and O'Carroll AM: Central and peripheral apelin receptor distribution in the mouse: species differences with rat. Peptides 33: 139-148, 2012.

9. Medhurst AD, Jennings CA, Robbins MJ, Davis RP, Ellis C, Winborn KY, Lawrie KW, Hervieu G, Riley G, Bolaky JE, et al: Pharmacological and immunohistochemical characterization of the APJ receptor and its endogenous ligand apelin. $\mathrm{J}$ Neurochem 84: 1162-1172, 2003.

10. Ishida J, Hashimoto T, Hashimoto Y, Nishiwaki S, Iguchi T, Harada S, Sugaya T, Matsuzaki H, Yamamoto R, Shiota N, et al: Regulatory roles for APJ, a seven-transmembrane receptor related to angiotensin-type 1 receptor in blood pressure in vivo. J Biol Chem 279: 26274-26279, 2004.

11. Szokodi I, Tavi P, Földes G, Voutilainen-Myllylä S, Ilves M, Tokola H, Pikkarainen S, Piuhola J, Rysä J, Tóth M and Ruskoaho H: Apelin, the novel endogenous ligand of the orphan receptor APJ, regulates cardiac contractility. Circ Res 91: 434-440, 2002.

12. Wang G, Anini Y, Wei W, Qi X, OCarroll AM, Mochizuki T, Wang HQ, Hellmich MR, Englander EW and Greeley GH Jr: Apelin, a new enteric peptide: localization in the gastrointestinal tract, ontogeny, and stimulation of gastric cell proliferation and of cholecystokinin secretion. Endocrinology 145: 1342-1348, 2004.

13. Castan-Laurell I, Dray C, Attane C, Duparc T, Knauf C and Valet P: Apelin, diabetes, and obesity. Endocrine 40: 1-9, 2011.

14. Lv D, Li L, Lu Q, Li Y, Xie F, Li H, Cao J, Liu M, Wu D, He L and Chen LX: PAK1-cofilin phosphorylation mediates human lung adenocarcinoma cells migration induced by apelin-13. Clin Exp Pharmacol Physiol (In Press).

15. Tiani C, Garcia-Pras E, Mejias M, de Gottardi A, Berzigotti A, Bosch J and Fernandez M: Apelin signaling modulates splanchnic angiogenesis and portosystemic collateral vessel formation in rats with portal hypertension. J Hepatol 50: 296-305, 2009.

16. Adam F, Khatib AM, Lopez JJ, Vatier C, Turpin S, Muscat A, Soulet F, Aries A, Jardin I, Bobe R, et al: Apelin: an antithrombotic factor that inhibits platelet function. Blood 127: 908-920, 2016.

17. Than A, Zhang X, Leow MK, Poh CL, Chong SK and Chen P. Apelin attenuates oxidative stress in human adipocytes. J Biol Chem 289: 3763-3774, 2014.

18. Foussal C, Lairez O, Calise D, Pathak A, Guilbeau-Frugier C Valet P, Parini A and Kunduzova O: Activation of catalase by apelin prevents oxidative stress-linked cardiac hypertrophy. FEBS Lett 584: 2363-2370, 2010.

19. Li L, Li F, Li F, Mao X, Yang L, Huang H, Guo Y, Chen L and Li J: NOX4-derived reactive oxygen species drive apelin-13-induced vascular smooth muscle cell proliferation via the ERK pathway. Int J Pept Res Ther 17: 307-315, 2011.

20. Katugampola SD, Maguire JJ, Matthewson SR and Davenport AP $[(125) \mathrm{I}]-(\mathrm{Pyr}(1))$ Apelin-13 is a novel radioligand for localizing the APJ orphan receptor in human and rat tissues with evidence for a vasoconstrictor role in man. Br J Pharmacol 132: 1255-1260, 2001.

21. De Falco M, De Luca L, Onori N, Cavallotti I, Artigiano F, Esposito V, De Luca B, Laforgia V, Groeger AM and De Luca A: Apelin expression in normal human tissues. In Vivo 16: 333-336, 2002.

22. Kleinz MJ and Davenport AP: Immunocytochemical localization of the endogenous vasoactive peptide apelin to human vascular and endocardial endothelial cells. Regul Pept 118: 119-125, 2004

23. Li F, Li L, Qin X, Pan W, Feng F, Chen F, Zhu B, Liao D, Tanowitz H, Albanese $\mathrm{C}$ and Chen L: Apelin-induced vascular smooth muscle cell proliferation: the regulation of cyclin D1. Front Biosci 13: 3786-3792, 2008

24. Liu C, Su T, Li F, Li L, Qin X, Pan W, Feng F, Chen F, Liao D and Chen L: PI3K/Akt signaling transduction pathway is involved in rat vascular smooth muscle cell proliferation induced by apelin-13 Acta Biochim Biophys Sin (Shanghai) 42: 396-402, 2010.
25. Mao XH, Tao S, Zhang XHui, Li F, Qin XP, Liao DF, Li LF and Chen LX: Apelin-13 promotes monocyte adhesion to human umbilical vein endothelial cell mediated by phosphatidylinositol 3-kinase signaling pathway. Prog Biochem Biophys 38: 1162-1170, 2011.

26. Lu Y, Zhu X, Liang GX, Cui RR, Liu Y, Wu SS, Liang QH, Liu GY, Jiang Y, Liao XB, et al: Apelin-APJ induces ICAM-1, VCAM-1 and MCP-1 expression via NF- $\mathrm{kB} / \mathrm{JNK}$ signal pathway in human umbilical vein endothelial cells. Amino Acids 43: 2125-2136, 2012

27. Lassègue $B$ and Clempus RE: Vascular NAD(P)H oxidases: Specific features, expression, and regulation. Am J Physiol Regul Integr Comp Physiol 285: R277-R297, 2003.

28. Potdar S and Kavdia M: NO/peroxynitrite dynamics of high glucose-exposed HUVECs: Chemiluminescent measurement and computational model. Microvasc Res 78: 191-198, 2009.

29. Cohen RA and Tong X: Vascular oxidative stress: The common link in hypertensive and diabetic vascular disease. J Cardiovasc Pharmacol 55: 308-316, 2010.

30. Liang JH, Li YN, Qi JS and Jia XX: Peroxynitrite-induced protein nitration is responsible for renal mitochondrial damage in diabetic rat. J Endocrinol Invest 33: 140-146, 2010.

31. Li L, Li L, Xie F, Zhang Z, Guo Y, Tang G, Lv D, Lu Q, Chen L and Li J: Jagged-1/Notch3 signaling transduction pathway is involved in apelin-13-induced vascular smooth muscle cells proliferation. Acta Biochim Biophys Sin (Shanghai) 45: 875-881, 2013.

32. Hashimoto T, Kihara M, Imai N, Yoshida S, Shimoyamada H, Yasuzaki H, Ishida J, Toya Y, Kiuchi Y, Hirawa N, et al: Requirement of apelin-apelin receptor system for oxidative stress-linked atherosclerosis. Am J Pathol 171: 1705-1712, 2007.

33. Leeper NJ, Tedesco MM, Kojima Y, Schultz GM, Kundu RK, Ashley EA, Tsao PS, Dalman RL and Quertermous T: Apelin prevents aortic aneurysm formation by inhibiting macrophage inflammation. Am J Physiol Heart Circ Physiol 296 : H1329-H1335, 2009.

34. Lv D, Li H and Chen L: Apelin and APJ, a novel critical factor and therapeutic target for atherosclerosis. Acta Biochim Biophys Sin (Shanghai) 45: 527-533, 2013

35. Lee DK, Saldivia VR, Nguyen T, Cheng R, George SR and O'Dowd BF: Modification of the terminal residue of apelin-13 antagonizes its hypotensive action. Endocrinology 146: 231-236, 2005.

36. Tatemoto K, Takayama K, Zou MX, Kumaki I, Zhang W, Kumano K and Fujimiya M: The novel peptide apelin lowers blood pressure via a nitric oxide-dependent mechanism. Regul Pept 99: 87-92, 2001.

37. Japp AG, Cruden NL, Barnes G, van Gemeren N, Mathews J, Adamson J, Johnston NR, Denvir MA, Megson IL, Flapan AD and Newby DE: Acute cardiovascular effects of apelin in humans: Potential role in patients with chronic heart failure. Circulation 121: 1818-1827, 2010.

38. Dikalova AE, Góngora MC, Harrison DG, Lambeth JD, Dikalov S and Griendling KK: Upregulation of Nox1 in vascular smooth muscle leads to impaired endothelium-dependent relaxation via eNOS uncoupling. Am J Physiol Heart Circ Physiol 299: H673-H679, 2010.

39. Ghiadoni L, Taddei S and Virdis A: Hypertension and endothelial dysfunction: Therapeutic approach. Curr Vasc Pharmacol 10: 42-60, 2012.

40. Siddiquee K,Hampton J,Khan S,Zadory D, Gleaves L, Vaughan DE and Smith LH: Apelin protects against angiotensin II-induced cardiovascular fibrosis and decreases plasminogen activator inhibitor type-1 production. J Hypertens 29: 724-731, 2011.

41. Sun X, Iida S, Yoshikawa A, Senbonmatsu R, Imanaka K, Maruyama K, Nishimura S, Inagami T and Senbonmatsu T: Non-activated APJ suppresses the angiotensin II type 1 receptor, whereas apelin-activated APJ acts conversely. Hypertens Res 34: 701-706, 2011.

42. Ryu S, Ornoy A, Samuni A, Zangen S and Kohen R: Oxidative stress in Cohen diabetic rat model by high-sucrose, low-copper diet: Inducing pancreatic damage and diabetes. Metabolism 57: 1253-1261, 2008

43. Kitada M, Kume S, Imaizumi N and Koya D: Resveratrol improves oxidative stress and protects against diabetic nephropathy through normalization of Mn-SOD dysfunction in AMPK/SIRT1-independent pathway. Diabetes 60: 634-643, 2011.

44. Lee SH, Nam BY, Kang EW, Han SH, Li JJ, Kim H, Kim SH, Kwak SJ, Park JT, Chang TI, et al: Effects of an oral adsorbent on oxidative stress and fibronectin expression in experimental diabetic nephropathy. Nephrol Dial Transplant 25: 2134-2141, 2010. 
45. Ha H, Yu MR, Choi YJ, Kitamura M and Lee HB: Role of high glucose-induced nuclear factor-kappaB activation in monocyte chemoattractant protein-1 expression by mesangial cells. J Am Soc Nephrol 13: 894-902, 2002.

46. Morii T, Fujita H, Narita T, Shimotomai T, Fujishima H, Yoshioka N, Imai $\mathrm{H}$, Kakei $\mathrm{M}$ and Ito S: Association of monocyte chemoattractant protein-1 with renal tubular damage in diabetic nephropathy. J Diabetes Complications 17: 11-15, 2003.

47. Day RT, Cavaglieri RC and Feliers D: Apelin retards the progression of diabetic nephropathy. Am J Physiol Renal Physiol 304: F788-F800, 2013.

48. Nishida M, Okumura Y, Oka T, Toiyama K, Ozawa S, Itoi $\mathrm{T}$ and Hamaoka $\mathrm{K}$ : The role of apelin on the alleviative effect of Angiotensin receptor blocker in unilateral ureteral obstruction-induced renal fibrosis. Nephron Extra 2: 39-47, 2012

49. Cameron NE and Cotter MA: Pro-inflammatory mechanisms in diabetic neuropathy: Focus on the nuclear factor kappa B pathway. Curr Drug Targets 9: 60-67, 2008.

50. Ganesh Yerra V, Negi G, Sharma SS and Kumar A: Potential therapeutic effects of the simultaneous targeting of the Nrf2 and NF- $\mathrm{KB}$ pathways in diabetic neuropathy. Redox Biol 1: 394-397, 2013.

51. Zeng XJ, Yu SP, Zhang L and Wei L: Neuroprotective effect of the endogenous neural peptide apelin in cultured mouse cortical neurons. Exp Cell Res 316: 1773-1783, 2010.

52. Simó R, Carrasco E, García-Ramírez M and Hernández C: Angiogenic and antiangiogenic factors in proliferative diabetic retinopathy. Curr Diabetes Rev 2: 71-98, 2006.

53. Tao Y, Lu Q, Jiang YR, Qian J, Wang JY, Gao L and Jonas JB: Apelin in plasma and vitreous and in fibrovascular retinal membranes of patients with proliferative diabetic retinopathy. Invest Ophthalmol Vis Sci 51: 4237-4242, 2010.

54. Lu Q, Feng J and Jiang YR: The role of apelin in the retina of diabetic rats. PLoS One 8: e69703, 2013.

55. Saint-Geniez M, Masri B, Malecaze F, Knibiehler B and Audigier Y: Expression of the murine msr/apj receptor and its ligand apelin is upregulated during formation of the retinal vessels. Mech Dev 110: 183-186, 2002.

56. Cain K, Bratton SB and Cohen GM: The Apaf-1 apoptosome: A large caspase-activating complex. Biochimie 84: 203-214, 2002.

57. Matsushita H, Morishita R, Nata T, Aoki M, Nakagami H, Taniyama Y, Yamamoto K, Higaki J, Yasufumi K and Ogihara T: Hypoxia-induced endothelial apoptosis through nuclear factorkappaB (NF-kappaB)-mediated bcl-2 suppression: In vivo evidence of the importance of NF-kappaB in endothelial cell regulation. Circ Res 86: 974-981, 2000.

58. Di Stilo A, Chegaev K, Lazzarato L, Fruttero R, Gasco A, Rastaldo R and Cappello S: Effects of nitric oxide donor antioxidants containing the phenol vitamin $\mathrm{E}$ substructure and a furoxan moiety on ischemia/reperfusion injury. Arzneimittelforschung 59: 111-116, 2009.

59. Rastaldo R, Cappello S, Folino A, Di Stilo A, Chegaev K, Tritto I, Pagliaro P and Losano G: Low concentrations of an nitric oxide-donor combined with a liposoluble antioxidant compound enhance protection against reperfusion injury in isolated rat hearts. J Physiol Pharmacol 61: 21-27, 2010.

60. Chen Z, Li T and Zhang B: Morphine postconditioning protects against reperfusion injury in the isolated rat hearts. J Surg Res 145: 287-294, 2008.

61. Zeng XJ, Zhang LK, Wang HX, Lu LQ, Ma LQ and Tang CS: Apelin protects heart against ischemia/reperfusion injury in rat. Peptides 30: 1144-1152, 2009.

62. Simpkin JC, Yellon DM, Davidson SM, Lim SY, Wynne AM and Smith CC: Apelin-13 and apelin-36 exhibit direct cardioprotective activity against ischemia-reperfusion injury. Basic Res Cardiol 102: 518-528, 2007

63. Sorli SC, Le Gonidec S, Knibiehler B and Audigier Y: Apelin is a potent activator of tumour neoangiogenesis. Oncogene 26 : 7692-7699, 2007

64. Han S, Wang G, Qi X, Lee HM, Englander EW and Greeley GH Jr: A possible role for hypoxia-induced apelin expression in enteric cell proliferation. Am J Physiol Regul Integr Comp Physiol 294: R1832-R1839, 2008.

65. Liu J and Wang Z: Increased oxidative stress as a selective anticancer therapy. Oxid Med Cell Longev 2015: 294303, 2015.

66. Sawicka E, Lisowska A, Kowal P and Długosz A: The role of oxidative stress in bladder cancer. Postepy Hig Med Dosw (Online) 69: 744-752, 2015 (In Polish).

67. Raina K, Tyagi A, Kumar D, Agarwal R and Agarwal C: Role of oxidative stress in cytotoxicity of grape seed extract in human bladder cancer cells. Food Chem Toxicol 61: 187-195. 2013.
68. Tong L, Chuang CC, Wu S and Zuo L: Reactive oxygen species in redox cancer therapy. Cancer Lett 367: 18-25, 2015.

69. Shipitsin M and Polyak K: The cancer stem cell hypothesis: In search of definitions, markers, and relevance. Lab Invest 88: 459-463, 2008

70. Reya T, Morrison SJ, Clarke MF and Weissman IL: Stem cells, cancer, and cancer stem cells. Nature 414: 105-111, 2001.

71. Shi X, Zhang Y, Zheng J and Pan J: Reactive oxygen species in cancer stem cells. Antioxid Redox Signal 16: 1215-1228, 2012.

72. Gu Q, Zhai L, Feng X, Chen J, Miao Z, Ren L, Qian X, Yu J, Li Y, Xu X, et al: Apelin-36, a potent peptide, protects against ischemic brain injury by activating the PI3K/Akt pathway. Neurochem Int 63: 535-540, 2013.

73. Silva A, Yunes JA, Cardoso BA, Martins LR, Jotta PY, Abecasis M, Nowill AE, Leslie NR, Cardoso AA and Barata JT: PTEN posttranslational inactivation and hyperactivation of the PI3K/Akt pathway sustain primary $\mathrm{T}$ cell leukemia viability. J Clin Invest : 3762-3774, 2008.

74. Min KJ, Lee JT, Joe EH and Kwon TK: An IкB $\alpha$ phosphorylation inhibitor induces heme oxygenase-1(HO-1) expression through the activation of reactive oxygen species (ROS)-Nrf2-ARE signaling and ROS-PI3K/Akt signaling in an NF- $\kappa \mathrm{B}$-independent mechanism. Cell Signal 23: 1505-1513, 2011.

75. Khaksari M, Aboutaleb N, Nasirinezhad F, Vakili A and Madjd Z: Apelin-13 protects the brain against ischemic reperfusion injury and cerebral edema in a transient model of focal cerebral ischemia. J Mol Neurosci 48: 201-208, 2012.

76. Bao HJ, Zhang L, Han WC and Dai DK: Apelin-13 attenuates traumatic brain injury-induced damage by suppressing autophagy. Neurochem Res 40: 89-97, 2015.

77. Kasai A, Kinjo T, Ishihara R, Sakai I, Ishimaru Y, Yoshioka Y, Yamamuro A, Ishige K, Ito Y and Maeda S: Apelin deficiency accelerates the progression of amyotrophic lateral sclerosis. PLoS One 6: e23968, 2011.

78. Kidoya H, Ueno M, Yamada Y, Mochizuki N, Nakata M, Yano T, Fujii R and Takakura N: Spatial and temporal role of the apelin/APJ system in the caliber size regulation of blood vessels during angiogenesis. EMBO J 27: 522-534, 2008.

79. Inuzuka H, Nishizawa H, Inagaki A, Suzuki M, Ota S, Miyamura H, Miyazaki J, Sekiya T, Kurahashi H and Udagawa Y: Decreased expression of apelin in placentas from severe preeclampsia patients. Hypertens Pregnancy 32: 410-421, 2013.

80. Bortoff KD, Qiu C, Runyon S, Williams MA and Maitra R: Decreased maternal plasma apelin concentrations in preeclampsia. Hypertens Pregnancy 31: 398-404, 2012.

81. Furuya M, Okuda M, Usui H, Takenouchi T, Kami D, Nozawa A, Shozu M, Umezawa A, Takahashi T and Aoki I: Expression of angiotensin II receptor-like 1 in the placentas of pregnancyinduced hypertension. Int J Gynecol Pathol 31: 227-235, 2012.

82. Vatish M, Randeva HS and Grammatopoulos DK: Hormonal regulation of placental nitric oxide and pathogenesis of preeclampsia. Trends Mol Med 12: 223-233, 2006.

83. Han S, Wang G, Qi X, Englander EW and Greeley GH Jr: Involvement of a Stat 3 binding site in inflammation-induced enteric apelin expression. Am J Physiol Gastrointest Liver Physiol 295: G1068-G1078, 2008.

84. Cai X, Chen X, Wang X, Xu C, Guo Q, Zhu L, Zhu S and Xu J: Pre-protective effect of lipoic acid on injury induced by $\mathrm{H}_{2} \mathrm{O}_{2}$ in IPEC-J2 cells. Mol Cell Biochem 378: 73-81, 2013.

85. Baregamian N, Song J, Jeschke MG, Evers BM and Chung DH: IGF-1 protects intestinal epithelial cells from oxidative stressinduced apoptosis. J Surg Res 136: 31-37, 2006.

86. Gad GI, Ismail RI, El-Masry SA and Gouda HR: Serum apelin in early-onset neonatal sepsis: Is it diagnostic? J Neonatal Perinatal Med 7: 207-212, 2014

87. Lesur O, Roussy JF, Chagnon F, Gallo-Payet N, Dumaine R, Sarret P, Chraibi A, Chouinard L and Hogue B: Proven infectionrelated sepsis induces a differential stress response early after ICU admission. Crit Care 14: R131, 2010.

88. Pan CS, Teng X, Zhang J, Cai Y, Zhao J, Wu W, Wang X, Tang CS and Qi YF: Apelin antagonizes myocardial impairment in sepsis. J Card Fail 16: 609-617, 2010

89. Mertens K, Lowes DA, Webster NR, Talib J, Hall L, Davies MJ, Beattie JH and Galley HF: Low zinc and selenium concentrations in sepsis are associated with oxidative damage and inflammation. Br J Anaesth 114: 990-999, 2015.

90. Bar-Or D, Carrick MM, Mains CW, Rael LT, Slone D and Brody EN: Sepsis, oxidative stress, and hypoxia: Are there clues to better treatment? Redox Rep 20: 193-197, 2015. 
91. Rastaldo R, Cappello S, Folino A and Losano G: Effect of apelinapelin receptor system in postischaemic myocardial protection: a pharmacological postconditioning tool? Antioxid Redox Signal 14: 909-922, 2011.

92. Azizi Y, Faghihi M, Imani A, Roghani M and Nazari A: Post-infarct treatment with [Pyrl]-apelin-13 reduces myocardial damage through reduction of oxidative injury and nitric oxide enhancement in the rat model of myocardial infarction. Peptides 46: 76-82, 2013.

93. Chen XY, Liu XM, Feng LL and Tang CS: Changes and clinical significance of serum Apelin in patients with severe sepsis and septic shock. Zhongguo Yi Xue Ke Xue Yuan Xue Bao 30: 131-135, 2008 (In Chinese)

94. Cao J, Li H and Chen L: Targeting drugs to APJ receptor: The prospect of treatment of hypertension and other cardiovascular diseases. Curr Drug Targets 16: 148-155, 2015.

95. Iturrioz X, Alvear-Perez R, De Mota N, Franchet C, Guillier F, Leroux V, Dabire H, Le Jouan M, Chabane H, Gerbier R, et al: Identification and pharmacological properties of E339-3D6, the first nonpeptidic apelin receptor agonist. FASEB J 24: 1506-1517, 2010.

96. Khan P, Maloney PR, Hedrick M, Gosalia P, Milewski M, Li L Roth GP, Sergienko E, Suyama E, Sugarman E, et al: Functional Agonists of the Apelin (APJ) Receptor. Probe Reports from the NIH Molecular Libraries Program [Internet]. Last Update: Dec 12, 2011.

97. Mendez M: Renin release: Role of SNAREs. Am J Physiol Regul Integr Comp Physiol 307: R484-R486, 2014.

98. Maloney PR, Khan P, Hedrick M, Gosalia P, Milewski M, Li L, Roth GP, Sergienko E, Suyama E, Sugarman E, et al: Discovery of 4-oxo-6-((pyrimidin-2-ylthio)methyl)-4H-pyran-3-yl 4-nitrobenzoate (ML221) as a functional antagonist of the apelin (APJ) receptor. Bioorg Med Chem Lett 22: 6656-6660, 2012.

99. Zhou N, Fang J, Acheampong E, Mukhtar M and Pomerantz RJ: Binding of ALX40-4C to APJ, a CNS-based receptor, inhibits its utilization as a co-receptor by HIV-1. Virology 312: 196-203, 2003.
100.Zhang Y, Maitra R, Harris DL, Dhungana S, Snyder R and Runyon SP: Identifying structural determinants of potency for analogs of apelin-13: Integration of C-terminal truncation with structure-activity. Bioorg Med Chem 22: 2992-2997, 2014.

101. Jin G, Yang P, Gong Y, Fan X, Tang J and Lin J: Effects of puerarin on expression of apelin and its receptor of $2 \mathrm{~K} 1 \mathrm{C}$ renal hypertension rats. Zhongguo Zhong Yao Za Zhi 34: 3263-3267, 2009 (In Chinese).

102. Wu D, He L and Chen L: Apelin/APJ system: A promising therapy target for hypertension. Mol Biol Rep 41: 6691-6703, 2014.

103. El Messari S, Iturrioz X, Fassot C, De Mota N, Roesch D and Llorens-Cortes C: Functional dissociation of apelin receptor signaling and endocytosis: Implications for the effects of apelin on arterial blood pressure. J Neurochem 90: 1290-1301, 2004.

104.Zhang BH, Wang W, Wang H, Yin J and Zeng XJ: Promoting effects of the adipokine, apelin, on diabetic nephropathy. PLoS One 8: e60457, 2013.

105. Chen H, Wan D, Wang L, Peng A, Xiao H, Petersen RB, Liu C, Zheng $\mathrm{L}$ and Huang $\mathrm{K}$ : Apelin protects against acute renal injury by inhibiting TGF- $\beta 1$. Biochim Biophys Acta 1852: 1278-1287, 2015.

106. Yang Y, Zhang X, Cui H, Zhang C, Zhu C and Li L: Apelin-13 protects the brain against ischemia/reperfusion injury through activating PI3K/Akt and ERK1/2 signaling pathways. Neurosci Lett 568: 44-49, 2014.

107. Xin Q, Cheng B, Pan Y, Liu H, Yang C, Chen J and Bai B: Neuroprotective effects of apelin-13 on experimental ischemic stroke through suppression of inflammation. Peptides 63: 55-62, 2015 